\title{
A Retrospective Multicenter Study of Carbon Ion Radiotherapy for Locally Advanced Olfactory Neuroblastomas
}

\author{
HIROAKI SUEFUJI ${ }^{1}$, MASASHI KOTO ${ }^{2}$, YUSUKE DEMIZU ${ }^{3}$, JUN-ICHI SAITOH ${ }^{4}$, \\ YOSHIYUKI SHIOYAMA ${ }^{1}$, HIROSHI TSUJI ${ }^{2}$, TOMOAKI OKIMOTO ${ }^{3}$, \\ TATSUYA OHNO ${ }^{4}$, KENJI NEMOTO ${ }^{5}$, TAKASHI NAKANO ${ }^{4}$ and TADASHI KAMADA ${ }^{2}$ \\ ${ }^{1}$ Ion Beam Therapy Center, SAGA-HIMAT Foundation, Tosu, Japan; \\ ${ }^{2}$ Hospital of the National Institute of Radiological Science, National Institutes for \\ Quantum and Radiological Sciences and Technology, Chiba, Japan; \\ ${ }^{3}$ Department of Radiology, Hyogo Ion Beam Medical Center, Tatsuno, Japan; \\ ${ }^{4}$ Gunma University Heavy Ion Medical Center, Maebashi, Japan; \\ ${ }^{5}$ Department of Radiation Oncology, Yamagata University Faculty of Medicine, Yamagata, Japan
}

\begin{abstract}
The purpose was to evaluate efficacy and safety of carbon ion radiotherapy $(C$-ion $R T$ ) in patients with locally advanced olfactory neuroblastomas (ONBs). This study was a sub-analysis of the Japan Carbon-Ion Radiation Oncology Study Group Study (1402 HN, UMIN000024473). Clinical data of T4 ONBs treated with C-ion RT at four Institutions between November 2003 and December 2014 were retrospectively reviewed. Twenty-one patients underwent C-ion RT. Seven patients had T4a and 14 had T4b tumours without cervical node metastases. The median follow-up period was 39 (range=5-111) months. The 3-year overall survival and local control rates were $88.4 \%$ and $83.0 \%$, respectively. Grade 4 late toxicity was observed in three patients, including ipsilateral optic nerve disorder $(n=2)$ and ipsilateral retinopathy $(n=1)$. C-Ion $R T$ is effective and can be a curative modality for T4 ONBs. Prospective multicenter studies are warranted to confirm these findings.
\end{abstract}

Olfactory neuroblastoma (ONB) of the head and neck is a rare malignant tumour that arises from the olfactory neuroepithelium in the upper nasal cavity with extension into the skull base, into the orbit and to the intracranial space (1, 2). Surgery is considered a curative treatment for localized

This article is freely accessible online.

Correspondence to: Hiroaki Suefuji, Ion Beam Therapy Center, SAGA-HIMAT Foundation, 3049 Harakoga-machi, Tosu, Saga 8410071, Japan. Tel: +81 942811897, Fax: +81 942811905, e-mail: sufg@saga-himat.jp

Key Words: Olfactory neuroblastoma, cancer of the head and neck, carbon ion radiotherapy, radiotherapy, multicenter study.
ONB, while postoperative radiotherapy (RT) is necessary for advanced ONBs. Definitive RT alone is considered insufficient therapy for ONBs (3). In advanced cases, intracranial extension may pose a surgical challenge (4). Surgical resection followed by postoperative RT is associated with reduced local recurrence (5).

Although ONB has been traditionally regarded as a radioresistant tumour, RT has widely been used as part of the treatment algorithm in adjuvant and definitive settings.

Compared to photons, carbon ions (C-ions) offer a higher linear energy transfer and greater relative biological effectiveness (RBE) and therefore provide a higher probability of tumour control. The physical characteristics of C-ions, such as their Bragg peak and small lateral scattering, are theoretically superior to those of photons in that $\mathrm{C}$-ions can allow a more localized delivery of the radiation dose.

This study is the first report for ONBs treated by C-ion RT. $\mathrm{C}$-Ion RT for various head and neck radioresistant malignancies has shown excellent results. Jingu et al. reported clinical results of 37 patients with malignant melanoma of the head and neck who received $\mathrm{C}$-ion RT with concurrent chemotherapy. The 3year local control (LC) and overall survival (OS) rates were $81.1 \%$ and $65.3 \%$, respectively (6). Koto et al. reported 3-year LC and OS rates of $76.9 \%$ and $59.1 \%$, respectively, in 22 patients with locally advanced sinonasal adenocarcinoma + reated with C-ion RT (7). Therefore, C-ion RT might be a useful and potentially curative option for unresectable head and neck tumours, including ONBs.

In November 2003, following a clinical trial, the Ministry of Health, Labour and Welfare in Japan approved C-ion RT as a highly advanced medical technology. As of the end of 2014, there were four C-ion facilities functioning in Japan (Hospital of the National Institute of Radiological Sciences, Hyogo Ion Beam Medical Center, Gunma University Heavy 
Ion Medical Center and the SAGA-HIMAT Foundation). We conducted a retrospective multicentre study to assess the clinical outcomes of $\mathrm{C}$-ion $\mathrm{RT}$ for head and neck malignancies [Japan Carbon-Ion Radiation Oncology Study Group (J-CROS) study: 1402 HN, UMIN000024473]. In this article, we report the results pertaining to a subgroup of patients with locally advanced T4 ONBs.

\section{Patients and Methods}

Eligibility. J-CROS $1402 \mathrm{HN}$ was a retrospective multicentre study conducted across four C-ion facilities in Japan. Patients provided informed consent for use of their personal information for research purposes. This study was approved by the Institutional Review Boards at each of the participant institutes and was carried out in accordance with the Declaration of Helsinki.

Patients with head and neck malignancies, including ophthalmic tumours, who received C-ion RT between November 2003 and December 2014 were included (UMIN000024473). The inclusion criteria were as follows: (a) histologically-confirmed malignancy, (b) no bone or soft-tissue tumours, (c) N0/1 M0 status, (d) medically inoperable tumours or refusal for surgery by the patient, (e) definitive intent, (f) measurable tumours, and (g) Eastern Cooperative Oncology Group performance status of 0-2 (8). Patients who had previously undergone irradiation for the same lesion were excluded. TNM classification of tumours was performed according to the seventh edition of the International Union Against Cancer (UICC) (9).

Nine-hundred and eight patients were enrolled in total. Of them, 21 patients with T4 ONBs (T4a: 7 and T4b: 14) were included in this analysis.

Evaluation of clinical outcome. LC was defined as no evidence of tumour regrowth in the planning target volume (PTV), including in the marginal region of the PTV. Regional control was defined as no evidence of regional lymph node recurrence or head and neck mucosal skip lesions outside the PTV. In normal tissues, acute and late reactions after $\mathrm{C}$-ion $\mathrm{RT}$ were reclassified according to the National Cancer Institute Common Terminology Criteria for Adverse Events, version 4.0 (10).

Statistical analyses. All survival times were calculated from the first day of C-ion RT. LC, OS, and cause-specific survival (CSS) rates were determined using the Kaplan-Meier method. Statistical analyses were performed using SPSS software, version 23 (IBM Corp., Armonk, NY, USA).

\section{Results}

Cohort characteristics. The patient and tumour characteristics for 21 patients are shown in Table I. All patients were re-staged according to the seventh edition of the Tumour-node-metastasis staging system of the UICC (9). Disease status in all 21 patients was classified as NOM0.

Treatment characteristics. C-Ion doses are expressed as photon-equivalent doses in Grays (Gy) (RBE) and were defined as the physical dose multiplied by the C-ion RBE. The biological flatness of the spread-out Bragg peak (SOBP)
Table I. Patients and tumour characteristics.

\begin{tabular}{llc}
\hline Number of patients & & 21 \\
Gender & Male/female & $16 / 5$ \\
Age, years & Median (range) & $53(39-78)$ \\
Performance status & $0 / 1$ & $10 / 11$ \\
Tumour classification & T4a/T4b & $7 / 14$ \\
Node classification & N0/N1 & $21 / 0$ \\
Tumour status & Naïve/recurrence & $17 / 4$ \\
Gross tumour volume $(\mathrm{cc})$ & Median (range) & $37.9(7.7-89.2)$ \\
\hline
\end{tabular}

Table II. Treatment characteristics.

\begin{tabular}{lc}
\hline Carbon ion radiotherapy & No. of patients \\
\hline Fractionation & \\
16 Fractions at 4 per week & 14 \\
26 Fractions at 5 per week & 7 \\
Dose and fractionation & 1 \\
$57.6 \mathrm{~Gy}(\mathrm{RBE}) / 16$ fractions & 5 \\
$64.0 \mathrm{~Gy}(\mathrm{RBE}) / 16$ fractions & 8 \\
$60.8 \mathrm{~Gy}(\mathrm{RBE}) / 16$ fractions & 6 \\
$65.0 \mathrm{~Gy}(\mathrm{RBE}) / 26$ fractions & 1 \\
$70.2 \mathrm{~Gy}(\mathrm{RBE}) / 26$ fractions & 5 \\
Median number of portals &
\end{tabular}

RBE, Relative biological effectiveness.

was normalized using the surviving fraction of human salivary gland tumour cells at the distal SOBP region where the carbon ion RBE was assumed to be 3 (11).

Treatment characteristics of C-ion RT in all patients are shown in Table II. Schedule selection was dependent on the institution and not on the patient. The target reference point dose was defined as the isocenter, and the PTV encompassed the minimally $90 \%$ dose line of the reference point dose. None of the patients were administered prophylactic neck irradiation.

Neoadjuvant chemotherapy was administered to four patients: ifosfamide, cisplatin and etoposide for two patients; cisplatin and etoposide for one patient; and uncertain agent(s) in the case of one patient. Three patients with recurrence and one newly diagnosed patient had received chemotherapy prior to $\mathrm{C}$-ion RT. None of the patients had received adjuvant chemotherapy.

LC and survival. The median follow-up period was 39 (range=5-111) months. Three patients developed local recurrence within the PTV. The 3-year LC rate was $83 \%$ (Figure 1). The LC rate was not significantly correlated with the gross tumor volume (GTV) and C-ion RT dose. As of last follow-up, two patients had died of ONB and two of 


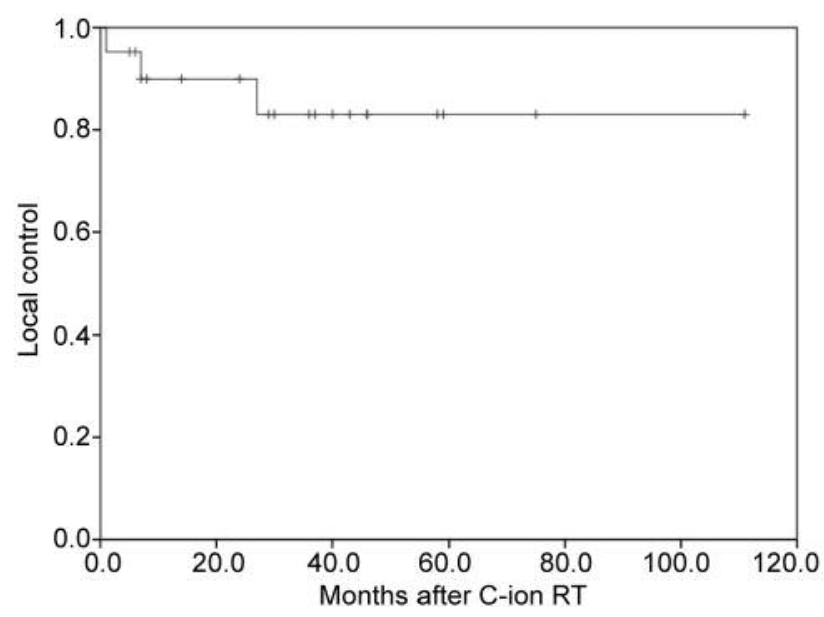

Figure 1. Local control rate after carbon ion radiotherapy $(C$-ion $R T)$ in patients with locally advanced olfactory neuroblastoma $(n=21)$. Both 3- and 5-year local control rates were $83.0 \%$.

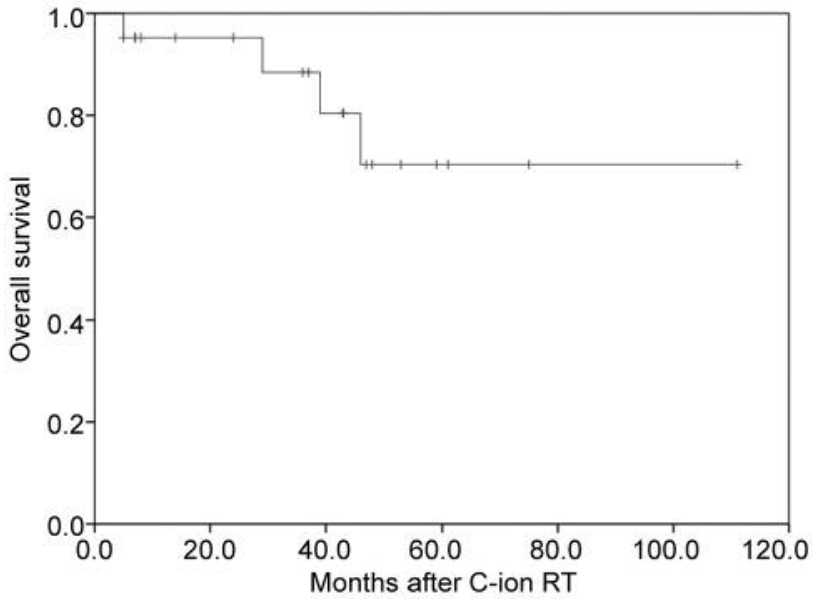

Figure 2. Overall survival after carbon ion radiotherapy $(C$-ion $R T)$ in patients with locally advanced olfactory neuroblastoma $(n=21)$. The 3 and 5-year survival rates were $88.4 \%$ and $70.3 \%$, respectively.

Table III. The number of late toxicity (grade 2 or more).

\begin{tabular}{|c|c|c|c|c|}
\hline \multirow[b]{2}{*}{ Toxicity } & \multicolumn{3}{|c|}{ Grade } & \multirow[t]{2}{*}{ Total } \\
\hline & 2 & 3 & 4 & \\
\hline Optic nerve disorder & 1 & 0 & 2 & 3 \\
\hline Retinopathy & 0 & 1 & 1 & 2 \\
\hline Watering eyes (due to obstruction of the nasolacrimal duct) & 1 & 0 & 0 & 1 \\
\hline Mucositis & 1 & 0 & 0 & 1 \\
\hline Soft tissue necrosis & 1 & 0 & 0 & 1 \\
\hline Osteonecrosis & 1 & 0 & 0 & 1 \\
\hline Central nerve system necrosis & 1 & 0 & 0 & 1 \\
\hline
\end{tabular}

unrelated causes. Six patients developed distant or nodal metastases (bone in two patients; cervical nodes in two patients; lung in one patient; data not available in one patient). The 3-year OS and CSS rates were $88.4 \%$ and $88.4 \%$, respectively (Figure 2 ).

Acute and late toxicity to normal tissues. A total of four patients developed grade 3 or higher C-ion RT-related acute toxicity. Grade 3 mucositis was observed in two patients. None of the patients experienced grade 4 or higher acute toxicity. The late toxicities are shown in Table III. With regard to visual function, three patients developed ipsilateral blindness (grade 4) and one developed grade 3 ipsilateral visual impairment. The causes of blindness were optic neuropathy in two patients and retinopathy in one patient. The median period between the initiation of $\mathrm{C}$-ion RT and the occurrence of ipsilateral blindness was 16 (range=9-29) months. The GTV was 39.3 (range=34.9-62.6) $\mathrm{cc}$ in three patients with blindness, and the tumours were in close proximity to the orbit. Except for eye disorders, grade 3 or more late toxicity was not encountered.

\section{Discussion}

ONB is a very rare disease that has been consistently associated with poor outcomes. Given the rarity of ONB, most published series were retrospective and had a small number of patients (12-15). In our knowledge, prospective clinical outcomes of ONB have not been reported. Ozsahin et al. reported 5-year OS and LC rates of $64 \%$ and $70 \%$, respectively, with multimodality therapy including surgery, RT and chemotherapy for T1-T4 ONBs (16). McLean et al. reported 5-year OS rate of 58\% in an ONB population of 21 patients with multimodality therapy including surgery, RT 
Table IV. Treatment outcomes in recent reports.

\begin{tabular}{|c|c|c|c|c|c|c|c|c|c|}
\hline Author & Year & $\mathrm{N}$ & $\begin{array}{c}\text { Proportion of } \\
\text { advanced cases }\end{array}$ & Treatment modality & MFM & OS & $\mathrm{LC}$ & PFS & Late toxicity \\
\hline Gruber et al. [11) & 2002 & 28 & $57 \%$ Kadish C & $\mathrm{S}+\mathrm{RT}$ or $\mathrm{RT} \pm \mathrm{CTx}$ & 68 & $77 \%(5 y)$ & $71 \%(5 \mathrm{y})$ & $70 \%(5 \mathrm{y})$ & NA \\
\hline Macleen et al. (16) & 2007 & 21 & $57 \%$ Kadish C & $\mathrm{S}, \mathrm{S}+\mathrm{RT} \pm \mathrm{CT} x, \mathrm{RT} \pm \mathrm{CT} x$ & 47 & $58 \%(5 y)$ & $81 \%$ (total) & $59 \%(5 y)$ & NA \\
\hline Zafereo et al. (12) & 2008 & 18 & $\begin{array}{c}22 \% \text { T4 } \\
50 \% \text { Kadish C }\end{array}$ & $\mathrm{S}, \mathrm{S} \pm \mathrm{RT} \pm \mathrm{CT} x, \mathrm{RT}+\mathrm{CTx}$ & 71 & $63 \%(5 y)$ & $83 \%(5 y)$ & $62 \%(5 y)$ & $\begin{array}{l}\text { NA (Mucositis: } 5 \text {, } \\
\text { osteomyelitis: } 2 \text {, } \\
\text { facial cellulitis: } 2 \text {, } \\
\text { epiphora: } 1 \text { and } \\
\text { sinusitis: } 1 \text { ) }\end{array}$ \\
\hline Modesto et al. (13) & 2013 & 43 & $\begin{array}{l}58 \% \text { Modified } \\
\text { Kadish C/D }\end{array}$ & multimodality therapy & 77 & $65 \%(5 y)$ & $72 \%$ (total) & $57 \%(5 y)$ & NA \\
\hline Ozashin et al. (15) & 2010 & 77 & $34 \% \mathrm{~T} 4$ & $\begin{array}{l}\mathrm{S}, \mathrm{S}+\mathrm{RT}, \mathrm{RT} \pm \mathrm{CTx} \\
\text { or multimodality }\end{array}$ & 72 & $64 \%(5 y)$ & $70 \%(5 y)$ & $57 \%(5 y)$ & $\begin{array}{l}\geq \mathrm{G} 39 \% \text { (Retinopathy, } \\
\text { osteo-/brain necrosis) }\end{array}$ \\
\hline Mori et al. (17) & 2015 & 17 & $82 \%$ Kadish C & $\mathrm{RT} \pm \mathrm{S} \pm \mathrm{CTx}$ & 95 & $88 \%(5 y)$ & NA & $74 \%(5 y)$ & $\begin{array}{c}\geq \mathrm{G} 217.6 \% \\
\text { (Hypopituitarism, } \\
\text { eye disorder) }\end{array}$ \\
\hline Noh et al. (14) & 2011 & 19 & $\begin{array}{l}\text { 74\% Modified } \\
\text { Kadish C/D }\end{array}$ & $\mathrm{S} \pm \mathrm{RT}, \mathrm{RT} \pm \mathrm{CT} x$ & 27 & $73 \%(3 y)$ & $78 \%$ (total) & $64 \%(3 y)$ & $\begin{array}{l}\text { NA (Retinopathy and } \\
\text { radiation-induced } \\
\text { sarcoma: 1) }\end{array}$ \\
\hline Nishimura et al. (18 & 8)2007 & 14 & $50 \%$ Kadish C & Proton & 40 & $93 \%(5 y)$ & $84 \%(5 y)$ & $71 \%(5 y)$ & $\geq \mathrm{G} 3: 0 \%$ \\
\hline Present study & - & 21 & $100 \% \mathrm{~T} 4$ & C-Ion RT & 39 & $88.4 \%(3 y)$ & $83 \%(3 y)$ & $65.3 \%(3 y)$ & (See text) \\
\hline
\end{tabular}

CTx, Chemotherapy; G, grade; LC, local control; MFM, median follow-up in months; NA, not applicable; OS, overall survival; PFS, progressionfree survival; proton, proton beam radiotherapy; RT, radiotherapy; S, surgery; 3y/5y:3-year/5-year.

and chemotherapy (17). In the present study, all patients had T4 ONBs; the OS and LC at 3 and 5 years were $88.4 \%$ and $70.3 \%$, and $83.0 \%$ and $83.0 \%$, respectively. These OS and $\mathrm{LC}$ values in a population of inoperable T4 ONBs in our study seemed favourable to those achieved with multimodality therapy in populations comprising all stages of ONBs (Table IV).

The reference period for our study exceeded 10 years; advances in surgical techniques and extended use of anticancer treatment over this period may have influenced patient outcomes. Modalities of RT have also evolved from 2D simulation to computed tomographic simulation (3DCRT) and, more recently, to use of intensity modulated RT (IMRT) technique. Mori et al. reported excellent results (5-year OS: $88 \%$ and 5-year progression-free survival rate $74 \%$ ) with multimodality therapy used 3DCRT and IMRT (18). Nishimura et al. reported a 5-year OS rate of $93 \%$ and 5 -year LC rate of $84 \%$ with use of proton beam RT; however, half of all patients were operable state of Kadish A and $\mathrm{B}$, which precludes a direct comparison with our results (19). Both proton beam RT and multimodality using IMRT and 3DCRT were considered effective treatments for ONBs. C-Ion RT might be one of effective treatments for locally advanced ONBs.

To our knowledge, this is the first report on use C-ion RT for locally advanced ONBs. Currently, there is no consensus on C-ion dose and fractionation. This study was a retrospective multicentre study, therefore, dose fractionation schedules of C-ion RT were not unified. Approximately $70 \%$ of patients were treated with 57.6-64.0 Gy (RBE)/16 fractions, while the remaining patients were treated with 65.0 or $70.2 \mathrm{~Gy}(\mathrm{RBE}) / 26$ fractions. In the three patients that developed local recurrence, the prescribed doses were $60.8 \mathrm{~Gy}(\mathrm{RBE}) / 16$ fractions, $64.0 \mathrm{~Gy}$ (RBE)/16 fractions and $65.0 \mathrm{~Gy}(\mathrm{RBE}) / 26$ fractions, respectively. There seems to be no clear correlation between local recurrence and dosefractionation schedule. It is difficult to determine the optimal dose based on our data.

Nalavenkata et al. reported a high incidence of neck metastases (15.9\%) and significantly lower OS in patients with delayed neck disease (73.8\%) compared with that in the absence of delayed neck disease (91.8\%) (20). In contrast, other studies appear to suggest that prophylactic neck irradiation may not be necessary in node-negative patients $(15,21)$. Although cervical node irradiation was not prescribed in this study, two patients experienced ipsilateral cervical node metastasis ( 33 and 57 months after C-ion RT). After cervical node resection, these two patients were still alive. Although the small number of patients makes it difficult to draw any definitive conclusions, we believe that cervical node resection is important in the event of nodal metastasis after C-ion RT.

In a study by Noh et al., none of the 17 patients with ONB developed cervical node metastases with systemic 
chemotherapy in the absence of cervical RT (15). However, a recent meta-analysis indicated that chemotherapy did not accrue any additional survival benefit in patients with ONBs (5). In our study, four patients received neo-adjuvant chemotherapy. Of these, one patient was treatment- naïve and three patients had disease recurrence. The present study does not allow for a valid assessment of the usefulness of chemotherapy for ONBs.

A few reports have referred to late toxicities. Noh et al. reported grade 3 or higher late toxicities of retinopathy, osteonecrosis and brain necrosis after multimodality therapy (15). Mori et al. reported a $17.6 \%$ incidence of grade 2 or more late adverse events (hypopituitarism and eye disorders) with use of 3DCRT and IMRT in 17 patients with ONBs (18). In this study, grade 3 or higher late toxicities occurred in four patients. Of these, two developed grade 4 ipsilateral optic nerve disorder, one grade 4 ipsilateral retinopathy, and one grade 3 ipsilateral retinopathy. The GTV of the three patients who developed blindness was 39.3 (range=34.962.6) $\mathrm{cc}$ and was larger than the median GTV 37.9 (range= 7.7-89.2) cc of all patients. Regarding optic neuropathy, which was a major cause of visual impairment, it was reported that a maximum $\mathrm{C}$-ion RT dose of 52 to $57 \mathrm{~Gy}$ (RBE) to the optic nerve was a significant negative prognostic factor $(22,23)$. Anatomically, ONB develops from the olfactory epithelium and is in close proximity to the orbit. However, in cases where the tumor invades the orbit and is close to the optic nerve, it is difficult to reduce the C-ion RT dose to the optic nerve. In these patients, probability of visual impairment was predictable, and C-ion RT was performed only with sufficient informed consent prior to the treatment. Other than eye toxicity, none of the other patients developed grade 3 or higher late toxicity.

The present study had several limitations. Firstly, the number of enrolled patients was small due to the rarity of ONBs. Secondly, this study was performed using retrospective data; therefore, several different doses and fractionations of C-ion RT were included. Since April 2016, an integrated treatment schedule using 16 fractions over 4 weeks with a total dose of 57.6 or $64.0 \mathrm{~Gy}$ (RBE) has been applied in a multicenter prospective registry study of Japan. Thirdly, the present study could not use the Kadish classification (24) and the Dulguerov and Cakcaterra staging system (25). J-CROS 1402HN was designed as a retrospective multicentre study conducted across four $\mathrm{C}$-ion facilities in Japan. We re-classified a total of 908 patients with various head and neck malignant tumours according to the seventh edition of the UICC TNM classification (9). Kadish C stage corresponds to T3 and T4 stage of the Dulguerov and Cakcaterra staging system. T3 stage of the Dulguerov and Cakcaterra staging system indicates tumour invasion into the orbit or frontal fossa without dural invasion. It is further subdivided into $\mathrm{T} 4 \mathrm{a}$ and $\mathrm{T} 4 \mathrm{~b}$ stages by the seventh edition of the UICC TNM classification. T4 of the Dulguerov and Cakcaterra staging system corresponds to T4b by the seventh edition of the UICC TNM classification.

In conclusion, $\mathrm{C}$-ion $\mathrm{RT}$ is an effective and potentially curative option for locally advanced ONBs, as an alternative to current multimodality therapy. Prospective multicentre studies are warranted to confirm these findings.

\section{Conflict of Interests}

The Authors have no conflicts of interest directly relevant to the content of this article.

\section{References}

1 Broich G, Pagliari A and Ottaviani F: Esthesioneuroblastoma: a general review of the cases published since the discovery of the tumour in 1924. Anticancer Res 17: 2683-2706, 1997.

2 Dulguerov P, Allal AS and Calcaterra TC: Esthesioneuroblastoma: a meta-analysis and review. Lancet Oncol 2: 683-690, 2001.

3 Kane AJ, Sughrue ME, Rutkowski MJ, Aranda D, Mills SA, Buencamino R, Fang S, Barani IJ and Parsa AT: Posttreatment prognosis of patients with esthesioneuroblastoma. J Neurosurg 113: 340-351, 2010.

4 Papacharalampous GX, Vlastarakos PV, Chrysovergis A, Saravakos PK, Kotsis GP and Davilis DI: Olfactory neuroblastoma (esthesioneuroblastoma): towards minimally invasive surgery and multi-modality treatment strategies - an updated critical review of the current literature. J BUON 18 : 557-o63, 2013.

5 Fu TS, Monteiro E, Muhanna N, Goldstein DP, de Almeida JR: Comparison of outcomes for open versus endoscopic approaches for olfactory neuroblastoma: A systematic review and individual participant data meta-analysis. Head Neck 38: E2306-2316, 2016.

6 Jingu K, Kishimoto R, Mizoe JE, Hasegawa A, Bessho H, Tsuji $\mathrm{H}$, Kamada T, Yamada $\mathrm{S}$ and Tsujii $\mathrm{H}$ : Malignant mucosal melanoma treated with carbon ion radiotherapy with concurrent chemotherapy: prognostic value of pretreatment apparent diffusion coefficient (ADC). Radiother Oncol 98: 68-73, 2011.

7 Koto M, Hasegawa A, Takagi R, Sasahara G, Ikawa H, Mizoe JE, Jingu K, Tsujii H, Kamada T, Okamoto Y; Organizing Committee for the Working Group for Head-and-Neck Cancer. Feasibility of carbon ion radiotherapy for locally advanced sinonasal adenocarcinoma. Radiother Oncol 111: 25-29, 2014.

8 Oken MM, Creech RH, Tormey DC, Horton J, Davis TE, McFadden ET and Carbone PP: Toxicity and response criteria of the Eastern Cooperative Oncology Group. Am J ClinOncol 5: 649-655, 1982.

9 International Union Against Cancer (UICC). In: Sobin LH, Gospodarowicz MK, Wittekind $\mathrm{CH}$, editors. TNM Classicfication of Malignant Tumors, 7th edition. New York: Willey; 2009.

10 National Cancer Institute. Common Terminology Criteria for Adverse Events (CTCAE) v4.0. Available from: URL: https://ctep.cancer.gov/protocolDevelopment/electronic_applicati ons/ctc.htm\#ctc_40 (cited 14 Dec 2017) . 
11 Inaniwa T, Kanematsu N, Matsufuji N, Kanai T, Shirai T, Noda K, Tsuji H, Kamada T and Tsujii H: Reformulation of a clinicaldose system for carbon-ion radiotherapy treatment planning at the National Institute of Radiological Sciences, Japan. Phys Med Biol 60: 3271-3286, 2015.

12 Gruber G, Laedrach K, Baumert B, Caversaccio M, Raveh J and Greiner R: Esthesioneuroblastoma: irradiation alone and surgery alone are not enough. Int J Radiat Oncol Biol Phys 54: 486-491, 2002.

13 Zafereo ME, Fakhri S, Prayson R, Batra PS, Lee J, Lanza DC and Citardi MJ: Esthesioneuroblastoma: 25-year experience at a single institution. Otolaryngol Head Neck Surg 138: 452-458, 2008.

14 Modesto A, Blanchard P, Tao YG, Rives M, Janot F, Serrano E, Benlyazid A, Guigay J, Ferrand FR, Delord JP, Bourhis J and Daly-Schveitzer N: Multimodal treatment and long-term outcome of patients with esthesioneuroblastoma. Oral Oncol 49: 830-834, 2013.

15 Noh OK, Lee SW, Yoon SM, Kim SB, Kim SY, Kim CJ, Jo KJ, Choi EK, Song SY, Kim JH and Ahn SD: Radiotherapy for esthesioneuroblastoma: is elective nodal irradiation warranted in the multimodality treatment approach? Int J Radiat Oncol Biol Phys 79: 443-449, 2011.

16 Ozsahin M, Gruber G, Olszyk O, Karakoyun-Celik O and Pehlivan B, Azria D, Roelandts M, Kaanders JH, Cengiz M, Krengli $\mathrm{M}$, Matzinger $\mathrm{O}$ and Zouhair A: Outcome and prognostic factors in olfactory neuroblastoma: a rare cancer network study. Int J Radiat Oncol Biol Phys 78: 992-997, 2010.

17 McLean JN, Nunley SR, Klass C, Moore C, Müller S and Johnstone PA: Combined modality therapy of esthesioneuroblastoma. Otolaryngol Head Neck Surg 136: 998-1002, 2007.

18 Mori T, Onimaru R, Onodera S, Tsuchiya K, Yasuda K, Hatakeyama H, Kobayashi H, Terasaka S, Homma A and Shirato $\mathrm{H}$ : Olfactory neuroblastoma: the long-term outcome and late toxicity of multimodal therapy including radiotherapy based on treatment planning using computed tomography. Radiat Oncol 10: 88,2015 .
19 Nishimura H, Ogino T, Kawashima M, Nihei K, Arahira S, Onozawa M, Katsuta S and Nishio T: Proton-beam therapy for olfactory neuroblastoma. Int J Radiat Oncol Biol Phys 68: 758$762,2007$.

20 Nalavenkata SB, Sacks R, Adappa ND, Palmer JN, Purkey MT, Feldman MD, Schlosser RJ, Snyderman CH, Wang EW, Woodworth BA, Smee R, Havas TE, Gallagher R and Harvey RJ: Olfactory Neuroblastoma: Fate of the Neck - A Long-term Multicenter Retrospective Study. Otolaryngol Head Neck Surg 154: 383-389, 2016

21 Elkon D, Hightower SI, Lim ML, Cantrell RW, Constable WC. Esthesioneuroblastoma. Cancer 44: 1087-1094, 1979.

22 Demizu Y, Murakami M, Miyawaki D, Niwa Y, Akagi T, Sasaki R, Terashima K, Suga D, Kamae I and Hishikawa Y: Analysis of vision loss caused by radiation-induced optic neuropathy after particle therapy for head-and-neck and skull-base tumors adjacent to optic nerves. Int J Radiat Oncol Biol Phys 75: 14871492, 2009.

23 Hasegawa A, Mizoe JE, Mizota A and Tsujii H: Outcomes of visual acuity in carbon ion radiotherapy: analysis of dosevolume histograms and prognostic factors. Int J Radiat Oncol Biol Phys 64: 396-401, 2006.

24 Kadish S, Goodman M and Wang CC: Olfactory neuroblastoma. A clinical analysis of 17 cases. Cancer 37: 157-156, 1976.

25 Dulguerov $\mathrm{P}$ and Calcaterra T: Esthesioneuroblastoma: the UCLA experience 1970-1990. Laryngoscope 102: 843-849, 1992. 